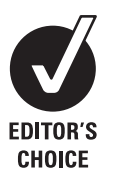

${ }^{1}$ School of Physiotherapy, Curtin University, Perth, Australia;

${ }^{2}$ Department of Physiotherapy Royal Perth Hospital, Perth, Australia; ${ }^{3}$ Australian Institute of Sport, Canberra, Australia

Correspondence to:

Associate Professor

Garry T Allison, Associate

Professor of Neuroscience and

Trauma Physiotherapy, School of

Physiotherapy, Curtin University,

Perth, Western Australia,

Physiotherapy Department,

Royal Perth Hospital Perth,

Western Australia; g.allison@

curtin.edu.au

Accepted 22 January 2009

Published Online First

11 February 2009

\title{
Eccentric loading for Achilles tendinopathy - strengthening or stretching?
}

\author{
G T Allison, ${ }^{1,2}$ C Purdam ${ }^{3}$
}

\section{ABSTRACT}

The prescription of eccentric loading is considered as a mainstay of non-operative rehabilitation programmes for mid-substance chronic Achilles tendinopathy. Such exercises have some degree of clinical utility in comparison with concentric training and are often referred to as a strengthening programme. Yet the dose parameters of the eccentric loading do not reflect an optimal strengthening programme and specifically avoid the normal eccentric-concentric coupling typical of the stretch shortening cycle. This manuscript identifies the arguments and counterarguments for why eccentric loading rehabilitation is not an optimal strengthening programme. It is proposed that such exercises reflect a specific stretching programme directed at the passive structures. This has two important clinical implications. Firstly, this reinterpretation of the role of eccentric exercises may direct future research into the underlying mechanisms of tendinopathy and, secondly, it may reinforce the importance of adjunct sports specific strengthening programmes.

In recent years the management of chronic Achilles tendinopathy (AT) has moved from addressing inflammatory responses (Achilles tendonitis) to exercise rehabilitation combined with pharmacological interventions. ${ }^{1-4}$ The non-operative clinical rehabilitation programmes for chronic AT are predominantly focused on progressive eccentric loading. ${ }^{1-5}$ Various studies have examined the impact of eccentric loading in comparison with other modalities including concentric exercises, splinting and stretching. A systematic review of the role of eccentric loading on AT shows it has some promise for the changes in pain. ${ }^{2}$ Direct comparison of eccentric and concentric programmes $^{6}$ suggests that eccentric exercises are more likely to generate a positive outcome (mean VAS change $18 \mathrm{~mm}, \mathrm{CI}-3.7$ to 39.7 ) in pain reduction than concentric exercises. Another recent systematic review shows that rehabilitation with eccentric exercises results in a greater relative rate of return to sport at 12 weeks (relative risk (RR) 2.38, CI 1.36 to 4.18) when compared with individuals who performed concentric exercises. ${ }^{4}$

Although it is acknowledged that further well controlled clinical trials are required, ${ }^{2}{ }^{4}$ these results have influenced clinical rehabilitation practice, with the prescription of eccentric loading for chronic AT being a mainstay of specific exercise therapy. Furthermore, certain prognostic factors are starting to be considered in the efficacy of the eccentric loading profiles with evidence that insertional pain and individuals who are more sedentary may not respond well to the standard eccentric loading protocol. That stated, there have been limited investigations of the training paradigm, i.e. frequency, dose and painful limits, with the majority of research reporting the specific exercise programme that follows the early work by Alfredson et $a l^{7}$ and Mafi et al. ${ }^{8}$ Representative programme parameters reported in comparative studies $^{7-9}$ are shown in table 1.

One key feature of the literature associated with eccentric loading is the reference to the eccentric programme as a progressive strength training programme. At face value, the benefits of the eccentric loading over the concentric resistance training may be explained by well known resistance training principles. These principles suggest that the eccentric actions are more specific, provide greater load via the force-velocity curve and generally provide a more effective resistance training programme. The eccentric loading profile described in the Achilles tendon literature, however, is not optimal for the production of muscle hypertrophy or contractile strength. Clearly, the eccentric-only programme is not specific to the stretch shortening cycle (SSC) nor is it maximally loading the tendon through the functional range (see table 2).

\section{More than just strengthening?}

Many rehabilitation therapists would argue that muscle performance deficits are seen in lower limbs with AT when compared with normal matched controls ${ }^{10}$ and increases in strength and endurance are observed following eccentric-only programmes. Yet, when eccentric exercises are compared with concentric exercises, the clinical improvements in symptoms and levels of function are clinically more evident than group differences in muscle performance. ${ }^{6}$ Therefore, it is plausible that the eccentric exercises have underlying mechanisms other than improvement in muscle performance. To further support this, table 3 provides the arguments that the eccentric programme used widely in the rehabilitation of the AT is far from an optimal muscle strengthening programme.

\section{Passive structure stretching programme? (Does the tendon know what the muscle fibre is doing?)}

When an external moment is applied to the joint, muscle fibres can create tension to resist the lengthening. This resisting force is transmitted through the tendon irrespective of the muscle fibres shortening (concentric) or lengthening (eccentric). Therefore one may ask "What is the mechanism that makes eccentric loading clinically 
Table 1 The eccentric loading programme parameters commonly used for the management of Achilles tendinopathy

\begin{tabular}{ll}
\hline $\begin{array}{l}\text { Exercise } \\
\text { parameter }\end{array}$ & Programme \\
\hline Repetitions & 15 \\
Sets & $\begin{array}{l}\text { Three of two similar exercises (see a and b below) } \\
\text { Excentric loading only, "good leg up bad leg down" } \\
\text { a) knee straight } \\
\text { b) knee bent }\end{array}$ \\
Frequency & $\begin{array}{l}\text { Twice daily, 7 days per week } \\
\text { Duration }\end{array}$ \\
Intensity & $\begin{array}{l}\text { Control parameter - discomfort/pain. Load to discomfort } \\
\text { (especially first 2 weeks). Increase load by external loading }\end{array}$ \\
Post-exercise & (Ice and stretch) \\
advice & \\
\hline From &
\end{tabular}

From $^{1378}$

effective compared with concentric muscle actions?" or, more simply, "How does the tendon (mid-substance Achilles) know what is happening to the muscle fibre?" This suggests that the eccentric load provides a different form of loading in the tendon and/or that there is communication between the muscle and the mid-substance tendon cells.

Recent research examining the different mechanical responses between eccentric and concentric loading may provide a mechanical basis that could explain either hypothesis. For example, Rees et a l $^{11}$ demonstrated that the peak tendon forces generated by the eccentric and concentric heel lowering and raising do not differ significantly. However, it was noted that during eccentric loading there was a greater degree of force oscillations within the tendon for the eccentric loading. It was proposed that this difference in the mechanical loading profile between the different muscle actions could explain, in part, the clinical efficacy. ${ }^{11}$

Potentially, the clinical efficacy could be a result of the interaction between the loading profile of the eccentric oscillating action and the range at which the tendon is loaded. With the loading occurring at relatively extreme dorsiflexion it is possible that the forces are predominantly absorbed by the passive structures as opposed to the muscle fibres. This is due to the fact that the muscle fibres are at a less than optimal length in the descending arm of the length-tension relationship. It may be this extreme range in combination with the loading that stimulates receptors in the aponeurosis to stimulate mechanical transduction along the length of the tendon. ${ }^{12}{ }^{13}$

Furthermore, the stretching of the tendon at the extreme range of motion may apply a different loading profile across different aspects of the tendon. ${ }^{14}$ It may be only during eccentric loading at the extreme ranges of motion that the specific source of the pathology is targeted. This may partly explain why tendinopathies with insertional pain may respond poorly to the "standard eccentric loading programme"15 and some suggest only lowering to ankle neutral in these specific cases. ${ }^{16}$

Perhaps it is the unique combination of ankle biomechanics and tendon load profile that explains the clinical efficacy of the eccentric loading. If both of these factors are important, one may question whether the generic "eccentric" programmes can be generalised to other tendons in other parts of the body. The evidence suggests that the eccentric loading profiles are clinically more efficacious at the ankle joint than at other joints, and therefore further understanding of the mechanisms is required to validate that eccentric exercise per se has a direct impact on all tendinopathies.
Table 2 Reasons and counterarguments for the superiority of eccentric exercises over concentric exercises for musculo-tendinous unit hypertrophy and strengthening in Achilles tendinopathy

Argument for Counterargument

Specificity

The tendo Achilles is loaded during The Achilles tendon is fundamental to lower limb the Stretch Shortening Cycle (SSC) SSC performance. The potentiation of the of running and jumping. The concentric phase of the SSC is a combination of eccentric loading regimen is specific to the SSC and optimises eccentric exercises as prescribed specifically the SSC through strengthening the avoid a concentric action following the eccentric SSC response. phase, thereby intentionally negating any concentric potentiation of the SSC. This may dampen the SSC response. Furthermore, the range at which peak loading occurs during these exercises is not specific for running and jumping.

Force-velocity advantages

The force-velocity curve clearly shows that greater loads can be achieved with eccentric loading than concentric. Therefore this explains why eccentric training has a benefit over the concentric loading regimen.

Eccentric actions generate a greater load on the musculo-tendinous unit than concentric actions at the same level of drive. There is no clear evidence that the tension in the tendon is any greater during the lowering process than an equivalent concentric action. ${ }^{11}$ The exercises have patients lower themselves from a plantar flexed position. To achieve a lowering the athlete must reduce the drive to the muscle substantially. Any increase in level of drive to the plantar flexors would actually slow the heel drop substantially and is the prime method of changing the velocity of the heel drop action.

\section{MECHANISMS}

If the premise that eccentric loading of the triceps surae is clearly not an optimal strengthening programme and it is acknowledged that it has some unique form of stretching stimulus, the question can be raised "What are the possible underlying mechanisms associated with changes seen in pathological Achilles tendons?" Some of the possible factors that may complement any strength or visco-elastic changes may include but are not limited to:

\section{Improved homogeneity of the passive structures}

It is possible that the pathology within the tendon reflects a heterogeneity in the visco-elastic properties of the tendon. The eccentric load may have a selective impact on how the tendon is loaded during the stretch. This is consistent with the so-called shielding hypothesis of tendon pathology. ${ }^{17}{ }^{18}$ The extreme range (dorsiflexion) and possibly the velocity or oscillations of the loading may also influence the heterogeneity of the tendon substance by providing a differential loading profile to different parts of the tendon. ${ }^{14}$ Furthermore, it may influence the connecting aponeurosis, which could influence a larger element of the whole musculo-tendinous unit. This in turn may alter the combination of the mechanical (elastic recoil) and neurological (spinal reflex) feedback loops required for optimal lower limb SSC function.

\section{Modulation of the neurological stretch responses}

The SSC relies on the synchronisation of both mechanical and neurological systems to optimise the concentric potentiation and overall movement efficiency. ${ }^{19}$ The pathogenesis or maintenance of AT may be a manifestation of a mismatch of the neurological and mechanical factors. Aggressive eccentric stretching (especially into pain) may cause peripheral and central neurological modulation of thresholds of activation, and synchronisation of motor units associated with short loop and long loop reflex contributions to the optimal SSC. ${ }^{20}$ 
Table 3 Arguments why the prescribed eccentric programme does not reflect a generic muscle hypertrophy strengthening programme

\begin{tabular}{|c|c|}
\hline $\begin{array}{l}\text { Programme details } \\
\text { (heel drops) }\end{array}$ & Generic strengthening hypertrophy \\
\hline Eccentric only & $\begin{array}{l}\text { Few strengthening programmes advocate eccentric-only } \\
\text { repetitions. When they do, this is often built on a foundation } \\
\text { of concentric training followed by a meso-cycle of so-called } \\
\text { "negatives". Secondly, eccentric loading may be used } \\
\text { specifically to induce changes in the peak force angle by } \\
\text { adding sarcomeres. }{ }^{23}\end{array}$ \\
\hline $\begin{array}{l}\text { Three sets of } 15 \text { twice } \\
\text { a day }\end{array}$ & $\begin{array}{l}\text { Hypertrophy programmes would normally utilise a lower } \\
\text { number of repetitions (commonly three sessions per week) } \\
\text { and would not prescribe sessions twice a day, } 7 \text { days a } \\
\text { week for } 10+\text { weeks. }\end{array}$ \\
\hline $\begin{array}{l}\text { Two similar exercises } \\
\text { (knees bent knees } \\
\text { straight) done in } \\
\text { sequence. (This is } \\
\text { sometimes described as } \\
\text { "supersets") }\end{array}$ & $\begin{array}{l}\text { Supersets are common in strength training programmes. } \\
\text { They are designed to fatigue one element of a muscle } \\
\text { synergy prior to performing more resistance training on the } \\
\text { whole movement pattern. The usual eccentric loading } \\
\text { programme does not require the sets to reach such levels of } \\
\text { fatigue. }\end{array}$ \\
\hline $\begin{array}{l}\text { Perform with some } \\
\text { discomfort }\end{array}$ & $\begin{array}{l}\text { Muscle hypertrophy-focused resistance training requires } \\
\text { maximal activation. Pain response from passive structures } \\
\text { such as tendon would not facilitate this type of training. }\end{array}$ \\
\hline Progress using velocity & $\begin{array}{l}\text { Increasing velocity of the eccentric drops is mostly reported } \\
\text { in early publications, }{ }^{5} \text { but if one uses velocity to increase } \\
\text { the load it is likely to reduce the drive to the plantar flexors } \\
\text { during the through-range movements. It is also likely to } \\
\text { increase the peak loading impact at end of range. Therefore } \\
\text { this training programme affects the end-range passive } \\
\text { structures rather than in mid-range where the muscle fibres } \\
\text { are able to generate resistance. }\end{array}$ \\
\hline
\end{tabular}

Furthermore, the eccentric loading may induce a change in the optimal position of the peak force production with the addition of muscle fibre sarcomeres. ${ }^{19}{ }^{22}{ }^{23}$ This could alter the relationship between the optimal functional ranges of the passive (tendon and aponeurosis) and active (muscle fibres) subsystems.

\section{Increased shear forces between the tendon and paratendon structures}

The eccentric loading profile has been shown to reduce vascularity as a result of the programme when the tendinopathy is associated with neovascularity. ${ }^{24}$ This is likely to be associated with the stresses generated within the tendon as well as the mechanical shear force stimulus between the tendon and the paratendon. These forces may inhibit, and possibly decrease, vascular infiltration into the tendon ${ }^{25}$ and alter the nociceptive inputs. This in turn may have secondary effects on the pain response and the ability to load the tendon.

\section{Other mechanisms}

There are many different aspects that warrant consideration as to why the eccentric loading systems seem to have a clinical advantage in AT. These include elements of pain production and desensitisation that may alter the reflex drive to the lower limb muscles. Another aspect gaining interest is the role of eccentric loading and the adaptation of mechanotransduction signalling in the passive tendon structures ${ }^{12}{ }^{13}$ and the proximal aponeurosis or muscle fibres. The fact that there are few well controlled trials associated with the actual dose of the intervention makes understanding the underlying mechanisms more difficult.

\section{CLINICAL SIGNIFICANCE}

One aspect that comes from this commentary is that the eccentric-only exercises that dominate the rehabilitation programmes for AT are not primarily a resistance training programme to increase muscle strength. Although we propose

\section{What is already known on this topic}

Eccentric heel drops have clinical utility for Achilles tendinopathy. There is growing research interest in the underlying mechanism that differentiates eccentric and concentric loading. Many clinicians perceive these exercises as strengthening exercises.

\section{What this study adds}

This study proposes that:

- the prescribed eccentric heel drops are not an optimal strengthening programme

- the exercises are more likely to generate a passive stretch

- in combination with eccentric loading, rehabilitation should incorporate sports-specific SSC and strengthening programmes

that the exercises could be more appropriately defined as a stretching programme, the main clinical message is that, in conjunction with this "stretching programme", well planned resistance training and a sports specific rehabilitation programme need to be considered. ${ }^{26}$ Sport-specific functional strengthening and endurance programmes should be used in parallel with the eccentric heel drop programme. Individuals who are not athletes, who have been shown to have poorer response rates, may benefit from a parallel programme of aerobic and lower limb resistance training. Few studies have examined the degree of compliance and other tendon-loading activities during activity of daily living in experimental paradigms. This does not mean that they are not important factors in clinical outcomes.

\section{SUMMARY}

The clinical utility of eccentric heel drops for chronic AT is not necessarily in response to an increase in strength of the plantar flexors, since this type of resistance training programme is less than optimal to achieve increases in strength. The reason why these exercises, when compared with other strengthening programmes, have greater clinical utility is unclear. There are possibilities that this type of loading profile has an additional impact specifically on the tendo Achilles due to its being an aggressive stretching programme suited to loading the passive structures. The clinical implication is that specific functional strengthening and endurance programmes should also be used in parallel to the eccentric programme. ${ }^{26}$

Specific reconditioning exercises that mimic the SSC and return to sport are also suggested to be critical in the efficacy of the stretching programme. These considerations should be part of an effort to investigate optimal combinations of stretching and specific strengthening programmes. Attempts to identify the importance of both strengthening and stretching elements in a rehabilitation programme may provide some insight into the underlying mechanism associated with AT and tendinopathies in general.

Acknowledgements: The physiotherapy staff and research students at the Australian Institute of Sport are acknowledged for their feedback on the original presentation of this work.

Funding: GTA was supported by a Research Fellowship funded by Club Warehouse at the Australian Institute of Sport Canberra.

Competing interests: None. 


\section{REFERENCES}

1. Alfredson $\mathbf{H}$, Cook JL. A treatment algorithm for managing Achilles tendinopathy: new treatment options. Br J Sports Med 2007;41:211-16.

2. Kingma JJ, de Knikker R, Wittink HM, et al. Eccentric overload training in patients with chronic Achilles tendinopathy: a systematic review. Br J Sports Med 2007; 41:e3.

3. Maffulli N, Khan KM, Puddu G. Overuse tendon conditions: time to change a confusing terminology. Arthroscopy 1998;14:840-3

4. Woodley BL, Newsham-West RJ, Baxter GD. Chronic tendinopathy: effectiveness of eccentric exercise. Br J Sports Med 2007;41:188-98; discussion 199.

5. Stanish WD, Rubinovich RM, Curwin S. Eccentric exercise in chronic tendinitis. Clin Orthop Relat Res 1986;208:65-8.

6. Silbernagel KG, Thomee R, Thomee $P$, et al. Eccentric overload training for patients with chronic Achilles tendon pain--a randomised controlled study with reliability testing of the evaluation methods. Scand J Med Sci Sports 2001;11:197-206.

7. Alfredson H, Pietila T, Jonsson P, et al. Heavy-load eccentric calf muscle training for the treatment of chronic Achilles tendinosis. Am J Sports Med 1998:26:360-6.

8. Mafi N, Lorentzon R, Alfredson H. Superior short-term results with eccentric calf muscle training compared to concentric training in a randomized prospective multicenter study on patients with chronic Achilles tendinosis. Knee Surg Sports Traumatol Arthrosc 2001;9:42-7.

9. Roos EM, Engstrom M, Lagerquist $A$, et al. Clinical improvement after 6 weeks of eccentric exercise in patients with mid-portion Achilles tendinopathy -- a randomized trial with 1-year follow-up. Scand J Med Sci Sports 2004;14:286-95.

10. Silbernagel KG, Gustavsson A, Thomee R, et al. Evaluation of lower leg function in patients with Achilles tendinopathy. Knee Surg Sports Traumatol Arthrosc 2006;14:1207-17.

11. Rees JD, Lichtwark GA, Wolman RL, et al. The mechanism for efficacy of eccentric loading in Achilles Tendon injury; an in vivo study in humans. Rheumatology 2008;47:1493-7.

12. Arnoczky SP, Lavagnino M, Egerbacher M, et al. Loss of homeostatic strain alters mechanostat "set point" of tendon cells in vitro. Clin Orthop Relat Res 2008:466:1583-91.
13. Egerbacher M, Arnoczky SP, Caballero 0, et al. Loss of homeostatic tension induces apoptosis in tendon cells: an in vitro study. Clin Orthop Relat Res 2008:466:1562-8.

14. Lyman J, Weinhold PS, Almekinders LC. Strain behavior of the distal achilles tendon: implications for insertional achilles tendinopathy. Am J Sports Med 2004:32:457-61.

15. Fahlstrom M, Jonsson P, Lorentzon R, et al. Chronic Achilles tendon pain treated with eccentric calf-muscle training. Knee Surg Sports Traumatol Arthrosc 2003:11:327-33.

16. Jonsson $\mathbf{P}$, Alfredson $\mathrm{H}$, Sunding $\mathrm{K}$, et al. New regimen for eccentric calf-muscle training in patients with chronic insertional Achilles tendinopathy: results of a pilot study. Br J Sports Med 2008;42:746-9.

17. Almekinders LC, Weinhold PS, Maffulli N. Compression etiology in tendinopathy, Clin Sports Med 2003;22:703-10.

18. Orchard JW, Cook JL, Halpin N. Stress-shielding as a cause of insertional tendinopathy: the operative technique of limited adductor tenotomy supports this theory. J Sci Med Sport 2004;7:424-8.

19. Morgan DL, Brockett CL, Gregory JE, et al. The role of the length-tension curve in the control of movement. Adv Exp Med Biol 2002;508:489-94.

20. Avela J, Komi PV. Reduced stretch reflex sensitivity and muscle stiffness after longlasting stretch-shortening cycle exercise in humans. Eur J Appl Physiol Occup Physiol 1998;78:403-10.

21. Nicol C, Avela J, Komi PV. The stretch-shortening cycle: a model to study naturally occurring neuromuscular fatigue. Sports Med 2006;36:977-99.

22. Brockett CL, Morgan DL, Proske U. Predicting hamstring strain injury in elite athletes. Med Sci Sports Exerc 2004;36:379-87.

23. Proske $\mathbf{U}$, Morgan DL, Brockett $\mathrm{CL}$, et al. Identifying athletes at risk of hamstring strains and how to protect them. Clin Exp Pharmacol Physiol 2004;31:546-50.

24. Ohberg L, Alfredson H. Effects on neovascularisation behind the good results with eccentric training in chronic mid-portion Achilles tendinosis? Knee Surg Sports Traumatol Arthrosc 2004;12:465-70.

25. Alfredson H. The chronic painful Achilles and patellar tendon: research on basic biology and treatment. Scand J Med Sci Sports 2005;15:252-9.

26. Kountouris A, Cook JL. Rehabilitation of Achilles and patellar tendinopathies. Best Pract Res Clin Rheumatol 2007:21:295-316. 